\section{A spinal scaffold}

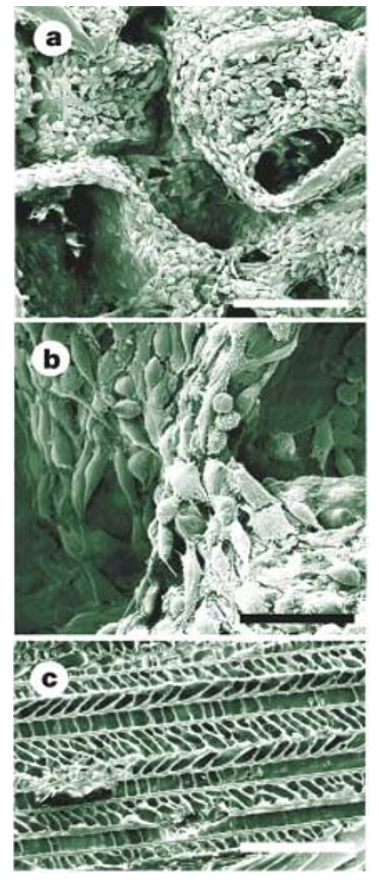

Over the past few years, we have witnessed the advent of many approaches that aim to promote functional recovery after injury to the spinal cord. Blockade of endogenous inhibitory molecules, myelin replacement by Schwann cells or olfactory ensheathing glia, and transplantation of embryonic tissue are just some of the strategies that have been developed and tested with varying degrees of success. A particularly intriguing approach is the implantation of biodegradable scaffolds into the injured region in the hope that they will promote recovery by fostering axonal growth or by supporting the survival of damaged cells. A recent paper by Teng et al.

Core of the scaffold seeded with neural stem cells (a,b). Outer portion of the scaffold, showing the axially oriented pores (c). ( 2002 National Academy of Sciences, USA. gives significant impetus to this idea by showing that a polymer scaffold that is seeded with neural stem cells promotes functional recovery in rats with spinal cord lesions.

The authors developed a scaffold, with a view to sustaining a structure akin to the spinal cord; it had a porous core that could be seeded with cells, and an outer portion with axially oriented pores that could potentially assist axon growth. They seeded scaffolds with neural stem cells, grafted them into longitudinal cavities in the spinal cords of rats, and tested whether this manipulation elicited behavioural recovery. The locomotion of animals that received cell-seeded scaffolds was better than the performance of rats that received cell-free scaffolds, which in turn was better than the locomotion of control rats or of animals that received only stem cells.

When they looked for the mechanisms that might underlie the behavioural recovery, the authors found that the presence of the scaf- fold diminished the formation of glial scars, leading them to suggest that this is one means by which the grafts might have a beneficial effect. Teng et al. also found evidence for axons crossing the lesion site in the presence of the scaffold, indicating that the recovery might also involve the regrowth of spared fibres.

Although it is still too early to herald this approach as the solution to spinal cord injury, the results of this study highlight the potential of bioengineering to help us develop innovative strategies to treat a variety of neurological and neurodegenerative disorders.

Juan Carlos López

\section{6) References and links}

ORIGINAL RESEARCH PAPER Teng, Y. D. et al. Functional recovery following traumatic spinal cord injury mediated by a unique polymer scaffold seeded with neural stem cells. Proc. Natl Acad. Sci. USA 99, 3024-3029 (2002)

FURTHER READING Raisman, G. Olfactory ensheathing cells - another miracle cure for spinal cord injury? Nature Rev. Neurosci. 2 , 369-374 (2001)

WEB SITES

Encyclopedia of Life Sciences:

http://www.els.net/

traumatic central nervous system injury

GLIOGENESIS

\title{
Oligodendro-sites
}

Oligodendrocytes are the myelinating cells of the central nervous system (CNS), and although they become widely distributed throughout the CNS, they seem to originate in a rather narrow zone in the neural tube. However, the precise location of this zone has been a matter of debate, with studies in rodent and chick embryos producing conflicting results. This has led some researchers to conclude that there are interspecies differences in the origin of oligodendrocytes. However, a new study by Fu et al. goes some way towards resolving the argument by showing that oligodendrocytes are in fact generated at two sites within the spinal cord.

The dorsoventral axis of the developing spinal cord is divided into several domains, each of which expresses a distinct combination of homeodomain proteins. Initial studies indicated that in the chick, oligodendrocyte progenitor cells (OLPs) arise in the $N k x 2.2$-expressing $\mathrm{p} 3$ domain, which generates $\mathrm{V} 3$ interneurons at an earlier stage of development. However, in the mouse, the OLPs seemed to originate in the more dorsal motor neuron precursor domain (pMN), which expresses the OLP marker Olig2. By examining the expression patterns of early oligodendrocyte markers, including Nkx2.2 and Olig2, the authors showed that, in fact, both zones could give rise to OLPs in both species. In addition, they found that the Olig2-expressing OLPs eventually acquire expression of $N k x 2.2$, although the timing of this event differs between species: chick OLPs already express both genes before they begin to migrate, whereas Olig2-expressing OLPs in the mouse, at least during the early phase of oligodendrogenesis, do not express $N k \times 2.2$ until they have left the proliferative zone. Also, in the chick embryo, $N k x 2$.2-expressing OLPs eventually acquire Olig2 expression, and in vitro studies indicate that this might also happen in mice.

Olig2 and Nkx2.2 co-expression in OLPs coincides with the onset of myelin gene expression in these cells, so Fu et al. investigated whether $N k x 2.2$ and Olig2 might have an instructive role in oligodendrocyte maturation. They showed that if either of the genes was inactivated using antisense oligonucleotides, myelin gene expression was decreased and the OLPs failed to undergo terminal differentiation. This indicates that $N k x 2.2$ and Olig2 might cooperate to induce oligodendrocyte differentiation.

This study shows that in both mouse and chick, OLPs are generated in two domains in the spinal cord neural tube, and that they need to express both $N k x 2.2$ and Olig2 to attain a mature oligodendrocyte phenotype. However, the timing of this co-expression, and of myelin gene activation, differs between the mouse and the chick, and it will be interesting to investigate the functional significance of this difference in relation to CNS maturation in these species.

Heather Wood

(10) References and links

ORIGINAL RESEARCH PAPER $\mathrm{Fu}$, $\mathrm{H}$ et al. Dual origin of spinal oligodendrocyte progenitors and evidence for the cooperative role of Olig2 and $N k \times 2.2$ in the control of oligodendrocyte differentiation. Development 129, 681-693 (2002)

FURTHER READING Marquardt, T. \& Pfaff, S. L. Cracking the transcriptional code for cell specification in the neural tube. Cell 106, 651-654 (2001) | Kessaris, N. et al. Ventral neurogenesis and the neuron-glial switch. Neuron $\mathbf{3 1}$, 677-680 (2001)

WEB SITES

Encyclopedia of Life Sciences: http://www.els.net oligodendrocytes 